\title{
PAPER
}

\section{Position representation of single-mode Gaussian channels beyond the Gaussian functional form}

To cite this article: David Davalos et al 2020 J. Phys. A: Math. Theor. 53425304

View the article online for updates and enhancements.

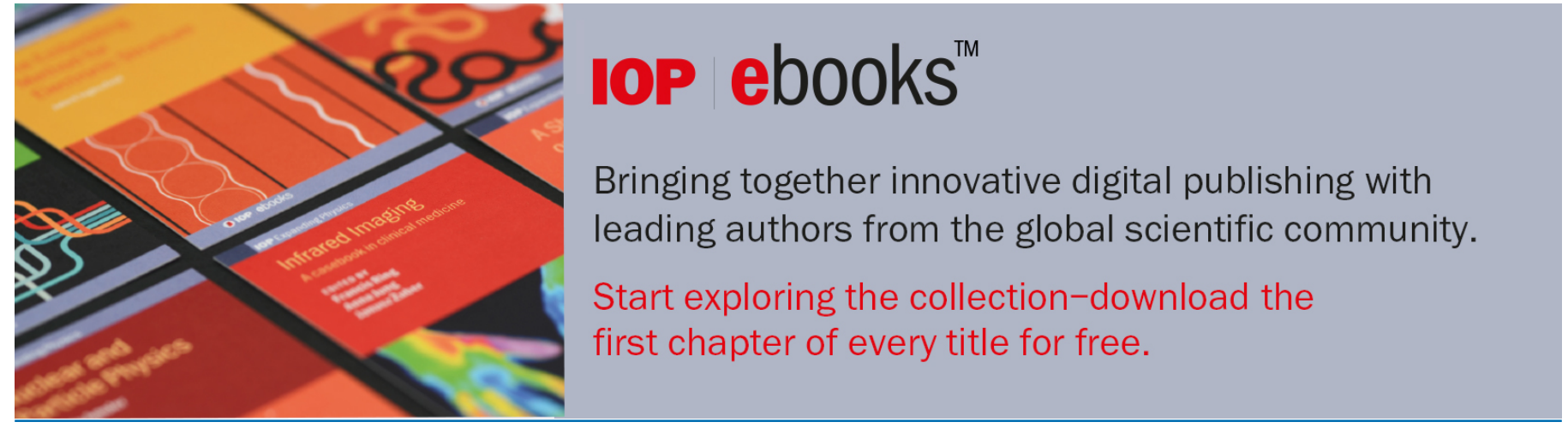

This content was downloaded from IP address 132.199 .102 .24 on $27 / 04 / 2021$ at $15: 13$ 


\title{
Position representation of single-mode Gaussian channels beyond the Gaussian functional form
}

\author{
David Davalos $^{1}{ }^{\circ}$, Camilo Moreno ${ }^{2}$, Juan-Diego Urbina ${ }^{2}$ \\ and Carlos Pineda ${ }^{1}$ (1) \\ ${ }^{1}$ Instituto de Física, Universidad Nacional Autónoma de México, Ciudad de México \\ 01000, México \\ ${ }^{2}$ Institut für Theoretische Physik, Universität Regensburg, D-93040 Regensburg, \\ Germany \\ E-mail: davidphysdavalos@gmail.com
}

Received 14 February 2020, revised 20 August 2020

Accepted for publication 24 August 2020

Published 1 October 2020

\begin{abstract}
We study one-mode Gaussian quantum channels in continuous-variable systems by performing a black-box characterization using complete positivity and trace preserving conditions, and report the existence of two subsets that do not have a functional Gaussian form. Our study covers as a particular limit the case of singular channels, thus connecting our results with their known classification scheme based on canonical forms. Our full characterization of Gaussian channels without Gaussian functional form is completed by showing how Gaussian states are transformed under these operations, and by deriving the conditions for the existence of master equations for the non-singular cases. We show that although every functional form can be found in the vicinity of the identity, one of them does not parametrize unitary channels.
\end{abstract}

Keywords: Gaussian quantum information, quantum information, continuous variable quantum information, singular Gaussian channels, quantum channels, Gaussian quantum channels

\section{Introduction}

Within the theory of continuous-variable quantum systems (a central topic of study given their role in the description of physical systems like the electromagnetic field [1], solids and nanomechanical systems [2] and atomic ensembles [3]) the simplest states, both from a theoretical an experimental point of view, are the so-called Gaussian states. An operation that transforms such family of states into itself is called a Gaussian quantum channel (GQC). Even though 
Gaussian states and channels form small subsets among general states and channels, they have proven to be useful in a variate of tasks such as quantum communication [4], quantum compu tation [5] and the study of quantum entanglement in simple [6] and complicated scenarios [7].

Writing Gaussian channels in the position state representation is often of theoretical convenience, for instance for the calculation of position correlation functions. Thus, an interesting way to proceed is to characterize the possible functional forms of GQC in such representation. First attempts in this direction were given in reference [8], but their ansatz is limited to only Gaussian functional forms (denoted simply by Gaussian forms or GF). Going beyond such restrictive assumption, in the present work we characterize another two possible forms that can arise directly from the definition of Gaussian channel in the one-mode case. We thus give a complete characterization of GQC in position state representation, and study the special case of singular Gaussian quantum channels (SGQC), i.e. the operations for which the inverse operation does not exist. There are works that study similar representation problems. For example, in reference [9] the authors studied the position state representation of Gaussian unitaries, suitable for close dynamics. We study Gaussian channels, which include Gaussian unitaries. However, our approach uses the quantum channel formalism (in the position state representation), which includes open system dynamics and allows for singular forms in contrast to reference [9]. In the same context of quantum channels, in reference [10] the authors studied the well known operator sum representation of Holevo's canonical forms, which characterize the action of one-mode Gaussian channels upto Gaussian unitaries [11]. On the other hand, our characterization goes over the full parametric space of one-mode Gaussian quantum channels, using the position state representation.

One surprising result of the present study is that key properties of Gaussian channels that contain delta-like factors cannot be reproduced by their smoothed form. This is particularly clear when considering binary properties like unitarity, as we will explicitly show.

The paper is organized as follows. In section 2 we discuss the definition of GQC and introduce functional forms beyond the GF that emerge from singularities in the coefficients that define a GQC with GF. In section 3 we give a black-box characterization of such channels, using complete positivity and trace preserving conditions. In section 4 we study functional forms that lead to SGQC and derive their explicit form. Finally in section 5 we derive conditions of existence of master equations and their explicit forms. We conclude in section 6.

\section{Gaussian quantum channels}

Gaussian states are characterized completely by first (mean) and second (correlations) moments encoded in the mean vector $\vec{d}$ and the covariance matrix $\sigma$. Therefore, a Gaussian state $S$ can be denoted as $S=S(\sigma, \vec{d})$, where for the one-mode case we have

$$
\sigma=\left(\begin{array}{cc}
\left\langle\hat{q}^{2}\right\rangle-\langle\hat{q}\rangle^{2} & \frac{1}{2}\langle\hat{q} \hat{p}+\hat{p} \hat{q}\rangle-\langle\hat{q}\rangle\langle\hat{p}\rangle \\
\frac{1}{2}\langle\hat{q} \hat{p}+\hat{p} \hat{q}\rangle-\langle\hat{q}\rangle\langle\hat{p}\rangle & \left\langle\hat{p}^{2}\right\rangle-\langle\hat{p}\rangle^{2}
\end{array}\right),
$$

and

$$
\vec{d}=(\langle\hat{q}\rangle,\langle\hat{p}\rangle)^{\mathrm{T}}
$$

with $\hat{q}$ and $\hat{p}$ denoting the standard position and momentum (quadrature) operators [12].

To start with, we recall the following definition [13]: 
Definition 1 (Gaussian quantum channels). A quantum channel is Gaussian (GQC) if it transforms Gaussian states into Gaussian states.

This definition is strictly equivalent to the statement that any GQC, say $\mathcal{G}$, can be written as

$$
\mathcal{G}[\rho]=\operatorname{tr}_{\mathrm{E}}\left[U\left(\rho \otimes \rho_{\mathrm{E}}\right) U^{\dagger}\right]
$$

where $U$ is a unitary transformation, acting on a combined global state obtained from enlarging the system with an environment $\mathrm{E}$, that is generated by a quadratic bosonic Hamiltonian (i.e. $U$ is a Gaussian unitary) [13]. The environmental initial state $\rho_{\mathrm{E}}$ is a Gaussian state and the trace is taken over the environmental degrees of freedom.

Following definition 1, a GQC is fully characterized by its action over Gaussian states, and this action is in turn defined by affine transformations [13]. Specifically, $\mathcal{G}=\mathcal{G}(\mathbf{T}, \mathbf{N}, \vec{\tau})$ is given by a tuple (T, $\mathbf{N}, \vec{\tau})$ where $\mathbf{T}$ and $\mathbf{N}$ are $2 \times 2$ real matrices with $\mathbf{N}=\mathbf{N}^{\mathrm{T}}$ [13] acting on Gaussian states according to $\mathcal{G}(\mathbf{T}, \mathbf{N}, \vec{\tau})[S(\sigma, \vec{d})]=S\left(\mathbf{T} \sigma \mathbf{T}^{\mathrm{T}}+\mathbf{N}, \mathbf{T} \vec{d}+\vec{\tau}\right)$. In the particular case of closed systems we have $\mathbf{N}=\mathbf{0}$ and $\mathbf{T}$ is a symplectic matrix, which corresponds to linear canonical transformations. Their unitary representations in the position state basis, together with their composition rules, was already studied in reference [9]. The authors showed that Gaussian unitaries have always a complex exponential form, while the superoperator representation of Gaussian unitaries (i.e. $\rho \mapsto U \rho U^{\dagger}$ ) does not (see lemma 1).

Let us note that although channels with Gaussian form trivially transform Gaussian states into Gaussian states, the definition goes beyond GF. Introducing difference and sum coordinates with the notation used in reference [14], $x=q_{2}-q_{1}$ and $r=\left(q_{1}+q_{2}\right) / 2$, such that $\rho(x, r)=\left\langle r-\frac{x}{2}|\hat{\rho}| r+\frac{x}{2}\right\rangle$, a quantum channel

$$
\rho_{\mathrm{f}}\left(x_{f}, r_{f}\right)=\int_{\mathbb{R}^{2}} \mathrm{~d} x_{i} \mathrm{~d} r_{i} J\left(x_{\mathrm{f}}, x_{\mathrm{i}} ; r_{\mathrm{f}}, r_{\mathrm{i}}\right) \rho_{\mathrm{i}}\left(r_{\mathrm{i}}, x_{\mathrm{i}}\right),
$$

maps an initial $\hat{\rho}_{\mathrm{i}}$ into a final $\hat{\rho}_{\mathrm{f}}$ state linearly through the kernel $J\left(x_{\mathrm{f}}, x_{\mathrm{i}} ; r_{\mathrm{f}}, r_{\mathrm{i}}\right)$. In order to see how a channel without GF can be constructed as a limiting case of a quantum channel with GF, consider the general parametrization of the latter as given in [15]

$$
\begin{aligned}
J_{\mathrm{G}}\left(x_{\mathrm{f}}, x_{\mathrm{i}} ; r_{\mathrm{f}}, r_{\mathrm{i}}\right)= & \frac{b_{3}}{2 \pi} \exp \left[\imath\left(b_{1} x_{\mathrm{f}} r_{\mathrm{f}}+b_{2} x_{\mathrm{f}} r_{\mathrm{i}}+b_{3} x_{\mathrm{i}} r_{\mathrm{f}}+b_{4} x_{\mathrm{i}} r_{\mathrm{i}}+c_{1} x_{\mathrm{f}}+c_{2} x_{\mathrm{i}}\right)\right. \\
& \left.-a_{1} x_{\mathrm{f}}^{2}-a_{2} x_{\mathrm{f}} x_{\mathrm{i}}-a_{3} x_{\mathrm{i}}^{2}\right],
\end{aligned}
$$

where all coefficients are real and no quadratic terms in $r_{\mathrm{i}, \mathrm{f}}$ are allowed due to the hermiticity and trace preserving conditions. Now it is easy to see that if the coefficients of the quadratic form in the exponent of $J_{\mathrm{G}}$ in equation (3) depend on a parameter $\epsilon$ such that for $\epsilon \rightarrow 0$ they scale as $a_{n} \propto \epsilon^{-1}$ and $b_{n} \propto \epsilon^{-1 / 2}$, then

$$
\lim _{\epsilon \rightarrow 0} J_{\mathrm{G}}\left(x_{\mathrm{f}}, x_{\mathrm{i}} ; r_{\mathrm{f}}, r_{\mathrm{i}}\right)=\mathcal{N} \delta\left(\alpha x_{\mathrm{f}}-\beta x_{\mathrm{i}}\right) \mathrm{e}^{\Sigma^{\prime}\left(x_{\mathrm{f}}, x_{\mathrm{i}}, r_{\mathrm{f}}, r_{\mathrm{i}}\right)},
$$

where $\alpha, \beta \in \mathbb{R}$ and $\Sigma^{\prime}\left(x_{\mathrm{f}}, x_{\mathrm{i}} ; r_{\mathrm{f}}, r_{\mathrm{i}}\right)$ is a quadratic form that now admits quadratic terms in $r_{\mathrm{i}, \mathrm{f}}$. This is the first example of a $\delta \mathrm{GQC}$, namely a Gaussian quantum channel that contains Diracdelta functions in its coordinate representation. This particular example is not only of academic interest. Physically, it can be implemented by means of the ubiquitous quantum Brownian motion (QBM) model for harmonic systems (damped harmonic oscillator) [14]. In such system $\delta \mathrm{GQC}$ occur at isolated points of time, defined in the limit of the antisymmetric position autocorrelation function tending to zero. 
Since the form of equation (4) admits quadratic terms in $r_{\mathrm{i}, \mathrm{f}}$, it suggest that a form with an additional delta can exist and can be defined with the usual limit of the Dirac delta using a Gaussian function. In order to avoid working with such limits, in this work we provide a black-box characterization of general GQCs without Gaussian form. In particular we study channels that can arise when singularities on the coefficients of Gaussian forms GF occur, that lead immediately to singular Gaussian operations. We characterize which forms in $\delta \mathrm{GQC}$ lead to valid quantum channels, and under which conditions singular operations lead to valid singular quantum channels (SGQC). We will show that only two possible forms of $\delta$ GQC hold according to trace preserving (TP) and hermiticity preserving (HP) conditions. The channel of equation (4) is one of these forms, as expected. Later on we will impose complete positivity in order to have valid GQC, i.e. completely positive and trace preserving (CPTP) Gaussian operations, going beyond previous characterizations of GQC in position state representation [15].

\section{Complete positive and trace-preserving $\delta$-Gaussian operations}

Let us introduce the ansätze for the possible forms of GQC in the position representation, to perform the black-box characterization. Following equation (1) and taking the continuous variable representation of difference and sum coordinates, the trace becomes an integral over position variables of the environment. Then we end up with a Fourier transform of a multivariate Gaussian, having for one mode the following structures: a Gaussian form equation (3), a Gaussian form multiplied with one-dimensional delta or a Gaussian form multiplied by a twodimensional delta. Thus, in order to start with the black-box characterization, we shall propose the following general Gaussian operations with one and two deltas, respectively

$$
\begin{aligned}
& J_{\mathrm{I}}\left(x_{\mathrm{f}}, r_{\mathrm{f}} ; x_{\mathrm{i}}, r_{\mathrm{i}}\right)=\mathcal{N}_{\mathrm{I}} \delta\left(\vec{\alpha}^{\mathrm{T}} \vec{v}_{\mathrm{f}}-\vec{\beta}^{\mathrm{T}} \vec{v}_{\mathrm{i}}\right) \mathrm{e}^{\sum\left(x_{\mathrm{f}}, x_{\mathrm{i}} ; r_{\mathrm{f}}, r_{\mathrm{i}}\right)} \\
& J_{\mathrm{II}}\left(x_{\mathrm{f}}, r_{\mathrm{f}} ; x_{\mathrm{i}}, r_{\mathrm{i}}\right)=\mathcal{N}_{\mathrm{II}} \delta\left(\mathbf{A} \vec{v}_{\mathrm{f}}-\mathbf{B} \vec{v}_{\mathrm{i}}\right) \mathrm{e}^{\sum\left(x_{\mathrm{f}}, x_{\mathrm{i}} ; r_{\mathrm{f}}, r_{\mathrm{i}}\right)}
\end{aligned}
$$

where the exponent reads

$$
\begin{aligned}
\Sigma\left(x_{\mathrm{f}}, x_{\mathrm{i}} ; r_{\mathrm{f}}, r_{\mathrm{i}}\right)= & \imath\left(b_{1} x_{\mathrm{f}} r_{\mathrm{f}}+b_{2} x_{\mathrm{f}} r_{\mathrm{i}}+b_{3} x_{\mathrm{i}} r_{\mathrm{f}}+b_{4} x_{\mathrm{i}} r_{\mathrm{i}}+c_{1} x_{\mathrm{f}}+c_{2} x_{\mathrm{i}}\right) \\
& -a_{1} x_{\mathrm{f}}^{2}-a_{2} x_{\mathrm{f}} x_{\mathrm{i}}-a_{3} x_{\mathrm{i}}^{2}-e_{1} r_{\mathrm{f}}^{2}-e_{2} r_{\mathrm{f}} r_{\mathrm{i}}-e_{3} r_{\mathrm{i}}^{2}-d_{1} r_{\mathrm{f}}-d_{2} r_{\mathrm{i}},
\end{aligned}
$$

with $\vec{v}_{\mathrm{i}(\mathrm{f})}=\left(r_{\mathrm{i}(\mathrm{f})}, x_{\mathrm{i}(\mathrm{f})}\right)$. A and $\mathbf{B}$ are $2 \times 2$ matrices, $\vec{\alpha}$ and $\vec{\beta}$ are two-dimensional vectors, and $\mathcal{N}_{\text {I,II }}$ are normalization constants. They provide, together with equation (3) all possible ansätze for GQC. Note that the coefficients in the exponential of every form must be finite, otherwise the functional form can be modified.

Let us study now CPTP conditions, since complete positivity implies positivity and in turn it implies hermiticity preserving (HP). For sum and difference coordinates HP reads

$$
J\left(-x_{\mathrm{f}}, r_{\mathrm{f}} ;-x_{\mathrm{i}}, r_{\mathrm{i}}\right)=J\left(x_{\mathrm{f}}, r_{\mathrm{f}} ; x_{\mathrm{i}}, r_{\mathrm{i}}\right)^{*}
$$

Following this equation, it is easy to note that the coefficients $a_{n}, b_{n}, c_{n}, e_{n}$ and $d_{n}$ must be real, as well the entries of matrices (and vectors) $\mathbf{A}, \mathbf{B}, \vec{\alpha}, \vec{\beta}$. The delta function in equation (5) is reduced to (i) $\delta\left(\alpha x_{\mathrm{f}}-\beta x_{\mathrm{i}}\right)$ or (ii) $\delta\left(\alpha r_{\mathrm{f}}-\beta r_{\mathrm{i}}\right)$ when fulfilling condition equation (8). For the case of equation (6), the two-dimensional delta is reduced to (iii) $\delta\left(\gamma r_{\mathrm{f}}-\eta r_{\mathrm{i}}\right) \delta\left(\alpha x_{\mathrm{f}}-\beta x_{\mathrm{i}}\right)$. Let us now analyze the trace preserving condition (TP), which for continuous variable systems reads 


$$
\int_{\mathbb{R}} \mathrm{d} r_{\mathrm{f}} J\left(x_{\mathrm{f}}=0, r_{\mathrm{f}} ; x_{\mathrm{i}}, r_{\mathrm{i}}\right)=\delta\left(x_{\mathrm{i}}\right)
$$

This condition immediately discards (ii) from the above combinations of deltas, thus we end up with cases (i) and (iii). For case (i) TP reads

$$
\mathcal{N}_{\mathrm{I}} \int \mathrm{d} r_{\mathrm{f}} \delta\left(-\beta x_{\mathrm{i}}\right) \mathrm{e}^{\Sigma}=\frac{\mathcal{N}_{\mathrm{I}}}{|\beta|} \sqrt{\frac{\pi}{e_{1}}} \delta\left(x_{\mathrm{i}}\right) \mathrm{e}^{\left(\frac{e_{2}^{2}}{4 e_{1}}-e_{3}\right) r_{\mathrm{i}}^{2}},
$$

thus the relation between the coefficients assumes the form

$$
\frac{e_{2}^{2}}{4 e_{1}}-e_{3}=0, \quad d_{1}=0, \quad d_{2}=0
$$

and the normalization constant $\mathcal{N}_{\mathrm{I}}=|\beta| \sqrt{\frac{e_{1}}{\pi}}$ with $\beta \neq 0$ and $e_{1}>0$. For case (iii) the tracepreserving condition reads

$$
\mathcal{N}_{\text {II }} \int \mathrm{d} r_{\mathrm{f}} \delta\left(\gamma r_{\mathrm{f}}-\eta r_{\mathrm{i}}\right) \delta\left(-\beta x_{\mathrm{i}}\right) \mathrm{e}^{\Sigma}=\frac{\mathcal{N}_{\mathrm{II}}}{|\beta \gamma|} \delta\left(x_{\mathrm{i}}\right) \mathrm{e}^{-\left(e_{1}\left(\frac{\eta}{\gamma}\right)^{2}+e_{2} \frac{\eta}{\gamma}+e_{3}\right) r_{\mathrm{i}}^{2}-\left(d_{1} \frac{\eta}{\gamma}+d_{2}\right) r_{\mathrm{i}}} .
$$

Thus, the following relation between $e_{n}$ and $d_{n}$ coefficients must be fulfilled:

$$
e_{1}\left(\frac{\eta}{\gamma}\right)^{2}+e_{2} \frac{\eta}{\gamma}+e_{3}=0, \quad d_{1} \frac{\eta}{\gamma}+d_{2}=0,
$$

with $\gamma, \beta \neq 0$ and $\mathcal{N}_{\text {II }}=|\beta \gamma|$. In the particular case of $\eta=0$, equation (12) is reduced to $e_{3}=d_{2}=0$. As expected from the analysis of limits above, we showed that $\delta$ GQCs admit quadratic terms in $r_{\mathrm{i}, \mathrm{j}}$.

Up to this point we have Hermitian and trace preserving Gaussian operations; to derive the remaining CPTP conditions, it is useful to write Wigner's function and Wigner's characteristic function, which we now derive. The representation of the Wigner's characteristic function reads

$$
\chi(\vec{k})=\exp \left[-\frac{1}{2} \vec{k}^{\mathrm{T}}\left(\Omega \sigma \Omega^{\mathrm{T}}\right) \vec{k}-\imath(\Omega\langle\hat{x}\rangle)^{\mathrm{T}} \vec{k}\right]
$$

and its relation with Wigner's function

$$
W(\mathbf{x})=\frac{1}{(2 \pi)^{2}} \int_{\mathbb{R}^{2}} \mathrm{~d} \vec{k} \mathrm{e}^{-\imath \vec{x}^{\mathrm{T}} \Omega \vec{k}} \chi(\vec{k})=\frac{1}{2 \pi} \int_{\mathbb{R}} \mathrm{d} x \mathrm{e}^{\imath p x}\left\langle r-\frac{x}{2}|\hat{\rho}| r+\frac{x}{2}\right\rangle .
$$

where $\vec{k}=\left(k_{1}, k_{2}\right)^{\mathrm{T}}, \vec{x}=(r, p)^{\mathrm{T}}$ and $\hbar=1$ (we are using natural units). Using the previous equations to construct Wigner and Wigner's characteristic functions of the initial and final states, and substituting them in equation (2), it is straightforward to get the propagator in the Wigner's characteristic function representation

$$
\tilde{J}\left(\vec{k}_{\mathrm{f}}, \vec{k}_{\mathrm{i}}\right)=\int_{\mathbb{R}^{6}} \mathrm{~d} \Gamma K(\vec{l}) J\left(\vec{v}_{\mathrm{f}}, \vec{v}_{\mathrm{i}}\right),
$$

where the transformation kernel reads

$$
K(\vec{l})=\frac{1}{(2 \pi)^{3}} \mathrm{e}^{\left[\imath\left(k_{2}^{\mathrm{f}} r_{\mathrm{f}}-k_{1}^{\mathrm{f}} p_{\mathrm{f}}-k_{2}^{\mathrm{i}} r_{\mathrm{i}}+k_{1}^{\mathrm{i}} p_{\mathrm{i}}-p_{\mathrm{i}} x_{\mathrm{i}}+p_{\mathrm{f}} x_{\mathrm{f}}\right)\right]}
$$


with

$$
\begin{gathered}
\mathrm{d} \Gamma=\mathrm{d} p_{\mathrm{f}} \mathrm{d} p_{\mathrm{i}} \mathrm{d} x_{\mathrm{f}} \mathrm{d} x_{\mathrm{i}} \mathrm{d} r_{\mathrm{f}} \mathrm{d} r_{\mathrm{i}} \text { and } \\
\vec{l}=\left(p_{\mathrm{f}}, p_{\mathrm{i}}, x_{\mathrm{f}}, x_{\mathrm{i}}, r_{\mathrm{f}}, r_{\mathrm{i}}\right)^{\mathrm{T}} .
\end{gathered}
$$

By elementary integration of equation (15) one can show that for both cases

$$
\tilde{J}_{\mathrm{I}, \mathrm{III}}\left(\vec{k}_{\mathrm{f}}, \vec{k}_{\mathrm{i}}\right)=\delta\left(k_{1}^{\mathrm{i}}-\frac{\alpha}{\beta} k_{1}^{\mathrm{f}}\right) \delta\left(k_{2}^{\mathrm{i}}-\vec{\phi}_{\mathrm{I}, \mathrm{TIII}}^{\mathrm{T}} \vec{k}_{\mathrm{f}}\right) \mathrm{e}^{\left.P_{\mathrm{I}, \mathrm{III}} \vec{k}_{\mathrm{f}}\right)},
$$

where $P_{\mathrm{I}, \mathrm{III}}\left(\vec{k}_{\mathrm{f}}\right)=\sum_{i, j=1}^{2} P_{i j}^{(\mathrm{I}, \mathrm{III})} k_{i}^{\mathrm{f}} k_{j}^{\mathrm{f}}+\sum_{i=1}^{2} P_{0 i}^{(\mathrm{I}, \mathrm{III})} k_{i}^{\mathrm{f}}$ with $P_{i j}^{(\mathrm{I}, \mathrm{III})}=P_{j i}^{(\mathrm{I}, \mathrm{III})}$. For case (i) we obtain

$$
\begin{aligned}
& P_{11}^{(\mathrm{I})}=-\left(\left(\frac{\alpha}{\beta}\right)^{2}\left(a_{3}+\frac{b_{3}^{2}}{4 e_{1}}\right)+\frac{\alpha}{\beta}\left(a_{2}+\frac{1}{2} \frac{b_{1} b_{3}}{e_{1}}\right)+a_{1}+\frac{b_{1}^{2}}{4 e_{1}}\right), \\
& P_{12}^{(\mathrm{I})}=-\left(\frac{\alpha}{\beta} \frac{b_{3}}{2 e_{1}}+\frac{b_{1}}{2 e_{1}}\right), \\
& P_{22}^{(\mathrm{I})}=-\frac{1}{4 e_{1}} .
\end{aligned}
$$

For case (iii) we have

$$
\begin{aligned}
& P_{11}^{(\mathrm{III})}=-\left(\left(\frac{\alpha}{\beta}\right)^{2} a_{3}+\frac{\alpha}{\beta} a_{2}+a_{1}\right), \\
& P_{12}^{(\mathrm{III})}=P_{22}^{(\mathrm{III})}=0,
\end{aligned}
$$

And for both cases we have $P_{01}^{(\mathrm{I}, \mathrm{III})}=\imath\left(\frac{\alpha}{\beta} c_{2}+c_{1}\right)$ and $P_{02}^{(\mathrm{I}, \mathrm{III})}=0$. Vectors $\vec{\phi}$ are given by

$$
\begin{aligned}
\vec{\phi}_{\mathrm{I}} & =\left(\frac{\alpha}{\beta}\left(b_{4}-\frac{b_{3} e_{2}}{2 e_{1}}\right)-\frac{b_{1} e_{2}}{2 e_{1}}+b_{2},-\frac{e_{2}}{2 e_{1}}\right)^{\mathrm{T}}, \\
\vec{\phi}_{\mathrm{III}} & =\left(\frac{\alpha}{\beta} \frac{\eta}{\gamma} b_{3}+\frac{\alpha}{\beta} b_{4}+\frac{\eta}{\gamma} b_{1}+b_{2}, \frac{\eta}{\gamma}\right)^{\mathrm{T}} .
\end{aligned}
$$

We are now in position to write explicitly the conditions for complete positivity. Having a Gaussian operation characterized by $(\mathbf{T}, \mathbf{N}, \vec{\tau})$, the CP condition can be expressed in terms of the matrix

$$
\mathbf{C}=\mathbf{N}+\imath \Omega-\imath \mathbf{T} \Omega \mathbf{T}^{\mathrm{T}},
$$

where $\Omega=\left(\begin{array}{cc}0 & 1 \\ -1 & 0\end{array}\right)$ is the symplectic matrix. An operation $\mathcal{G}(\mathbf{T}, \mathbf{N}, \vec{\tau})$ is $\mathrm{CP}$ if and only if $\mathbf{C} \geqslant 0[13,16]$. Applying the propagator on a test characteristic function, equation (13), it is easy to compute the corresponding tuples. For both cases we get $\left(\mathbf{T}_{\mathrm{I}, \mathrm{III}}, \mathbf{N}_{\mathrm{I}, \mathrm{III}}, \vec{\tau}_{\mathrm{I}, \mathrm{III}}\right)$ :

$$
\begin{aligned}
& \mathbf{N}_{\mathrm{I}, \mathrm{III}}=2\left(\begin{array}{cc}
-P_{22} & P_{12} \\
P_{12} & -P_{11}
\end{array}\right), \\
& \vec{\tau}_{\mathrm{I}, \mathrm{III}}=\left(0, \imath P_{01}^{(\mathrm{I}, \mathrm{III})}\right)^{\mathrm{T}},
\end{aligned}
$$


while for case (i) matrix $\mathbf{T}$ is given by

$$
\mathbf{T}_{\mathrm{I}}=\left(\begin{array}{cc}
\frac{e_{2}}{2 e_{1}} & 0 \\
\vec{\phi}_{\mathrm{I}, 1} & -\frac{\alpha}{\beta}
\end{array}\right),
$$

where $\vec{\phi}_{\mathrm{I}, 1}$ denotes the first component of vector $\vec{\phi}_{\mathrm{I}}$, see equation (19). The complete positive condition is given by the inequalities raised from the eigenvalues of matrix equation (20)

$$
\pm \frac{\sqrt{\alpha^{2} e_{2}^{2}+4 \alpha \beta e_{2} e_{1}+4 \beta^{2} e_{1}^{2}\left(4 P_{12}^{(\mathrm{I})^{2}}+\left(P_{11}^{(\mathrm{I})}-P_{22}^{(\mathrm{I})}\right)^{2}+1\right)}}{2 \beta e_{1}}-\left(P_{11}^{(\mathrm{I})}+P_{22}^{(\mathrm{I})}\right) \geqslant 0 .
$$

For case (iii) matrix $\mathbf{T}$ is

$$
\mathbf{T}_{\mathrm{III}}=\left(\begin{array}{cc}
-\frac{\eta}{\gamma} & 0 \\
\vec{\phi}_{\mathrm{III}, 1} & -\frac{\alpha}{\beta}
\end{array}\right),
$$

and complete positivity conditions read

$$
\pm \frac{\sqrt{\left.(\beta \gamma-\alpha \eta)^{2}+\beta^{2} \gamma^{2} P_{11}^{(\mathrm{III})}\right)^{2}}}{\beta \gamma}-P_{11}^{(\mathrm{III})} \geqslant 0 .
$$

Note that in both cases complete positivity conditions do not depend on $\vec{\phi}$. These results will be discussed in the next section for the singular case.

\section{Allowed singular forms}

There are two classes of Gaussian singular channels. Since the inverse of a Gaussian channel $\mathcal{G}(\mathbf{T}, \mathbf{N}, \vec{\tau})$ is $\mathcal{G}\left(\mathbf{T}^{-1},-\mathbf{T}^{-1} \mathbf{N} \mathbf{T}^{-T},-\mathbf{T}^{-1} \vec{\tau}\right)$, its existence rests on the invertibility of $\mathbf{T}$. Thus, studying the rank of the latter we are able to explore singular forms. We are going to use the classification of one-mode channels developed by Holevo [11].

For singular channels there are two classes characterized by its canonical form [17], i.e. any channel can be obtained by applying Gaussian unitaries before and after the canonical form. The class called ' $\mathrm{A}_{1}$ ' correspond to singular channels with $\operatorname{Rank}(\mathbf{T})=0$ and coincide with the family of total depolarizing channels. The class ' $\mathrm{A}_{2}$ ' is characterized by $\operatorname{Rank}(\mathbf{T})=1$. Both classes are entanglement-breaking [17].

Before analysing the functional forms constructed in this work, let us study channels with GF. The tuple of the affine transformation, corresponding to the propagator $J_{\mathrm{G}}$, equation (3), were introduced in reference [15] up to some typos. Our calculation for this tuple is

$$
\mathbf{T}_{\mathrm{G}}=\left(\begin{array}{cc}
-\frac{b_{4}}{b_{3}} & \frac{1}{b_{3}} \\
\frac{b_{1} b_{4}}{b_{3}}-b_{2} & -\frac{b_{1}}{b_{3}}
\end{array}\right)
$$




$$
\begin{aligned}
& \mathbf{N}_{\mathrm{G}}=\left(\begin{array}{cc}
\frac{2 a_{3}}{b_{3}^{2}} & \frac{a_{2}}{b_{3}}-\frac{2 a_{3} b_{1}}{b_{3}^{2}} \\
\frac{a_{2}}{b_{3}}-\frac{2 a_{3} b_{1}}{b_{3}^{2}} & -2\left(-\frac{a_{3} b_{1}^{2}}{b_{3}^{2}}+\frac{a_{2} b_{1}}{b_{3}}-a_{1}\right)
\end{array}\right), \\
& \vec{\tau}_{\mathrm{G}}=\left(-\frac{c_{2}}{b_{3}}, \frac{b_{1} c_{2}}{b_{3}}-c_{1}\right)^{\mathrm{T}} .
\end{aligned}
$$

It is straightforward to check that for $b_{2}=0, \mathbf{T}_{\mathrm{G}}$ is singular with $\operatorname{Rank}\left(\mathbf{T}_{\mathrm{G}}\right)=1$, i.e. it belongs to class $A_{2}$. Due to the full support of Gaussian functions, it was surprising that Gaussian channels with GF have singular limit. In this case the singular behavior arises from the lack of a Fourier factor for $x_{\mathrm{f}} r_{\mathrm{i}}$. This is the only singular case for GF.

It is instructive at this point to analyze the physical meaning of the transition from nonsingular to singular Gaussian channels in the context of QBM [14]. There, a central harmonic oscillator is linearly coupled through its position with a bath of harmonic oscillators initially at thermal equilibrium. The coupling of the central system to the bath causes dissipation of energy and decoherence in the position basis. At any time $t$ the quantum channel of QBM has the Gaussian form given by equation (3), with time-depending coefficients. Excluded here are particular times with divergent coefficients that need to be treated differently, as already mentioned in section 3 .

For a specific form of the bath spectral density all these coefficients are calculated in reference [14], and in particular the coefficient $b_{2}$ is found to be proportional to $\mathrm{e}^{-\gamma t / 2} \sin (\eta t)$, where $\gamma$ is the damping parameter characterizing the interaction strength and $\eta$ is a coefficient related with the natural frequency of the central oscillator. It is clear that for any finite $\gamma, b_{2} \rightarrow 0$ when $t \rightarrow \infty$, and then for long times the matrix $\mathbf{T}_{\mathrm{G}}$ becomes singular. It is precisely at $t \rightarrow \infty$ when the particle reaches the state of equilibrium in the QBM model (the reduced density matrix of the particle reaches a stationary state). Moreover, it can be shown that the quantum channel in the QBM model acquires the form $J_{\mathrm{I}}$, with $b_{2}=b_{1}=c_{1}=c_{2}=0$, which actually defines a singular channel, as shown above. Finally, it is easy to see that the determinant of the $\mathbf{T}_{\mathrm{G}}$ matrix in QBM is proportional to $\eta^{2} \mathrm{e}^{-\gamma t}$, and thus $\mathbf{T}_{\mathrm{G}}$ becomes singular only when $t \rightarrow \infty$. This shows the deep relation between dynamical irreversibility and singular channels in this context.

Now we analyze functional forms derived in section 3 . The complete positivity conditions of the form $\tilde{J}_{\text {III }}$, presented in equation (25), have no solution for $\alpha \rightarrow 0$ and/or $\gamma \rightarrow 0$, thus this form cannot lead to singular channels. This is not the case for $\tilde{J}_{\mathrm{I}}$, equation (16), which leads to singular operations belonging to class $\mathrm{A}_{2}$ for $\alpha e_{2}=0$, and to class $\mathrm{A}_{1}$ for $e_{2}=\alpha=b_{2}=0$. For the latter, the complete positivity conditions read

$$
e_{1} \leqslant a_{1}
$$

By using an initial state characterized by $\sigma_{\mathrm{i}}$ and $\vec{d}_{i}$ we can compute the explicit dependence of the final states on the initial parameters. For channels belonging to class $\mathrm{A}_{2}$ (see equation (26) with $b_{2}=0$ and equation (22) with $e_{2} \alpha=0$ ) the final state only depends in one combination of the components of $\sigma_{\mathrm{i}}$, and in one combination of the components of $\vec{d}_{i}$, i.e. $\sum_{m n} l_{m n}\left(\sigma_{i}\right)_{m n}$ and $\sum_{m} n_{m}\left(\vec{d}_{i}\right)_{m}$, respectively, where $l_{m n}$ and $n_{m}$ depend on the channel parameters. See the appendix for the explicit formulas and figure 1 (right panel) for an schematic description of the final states. From such combinations it is obvious that we cannot solve for the initial state parameters given a final state as expected; this is because the parametric space dimension is reduced from 5 to 2 . The channel belonging to $\mathrm{A}_{1}$ (see equation (22) 


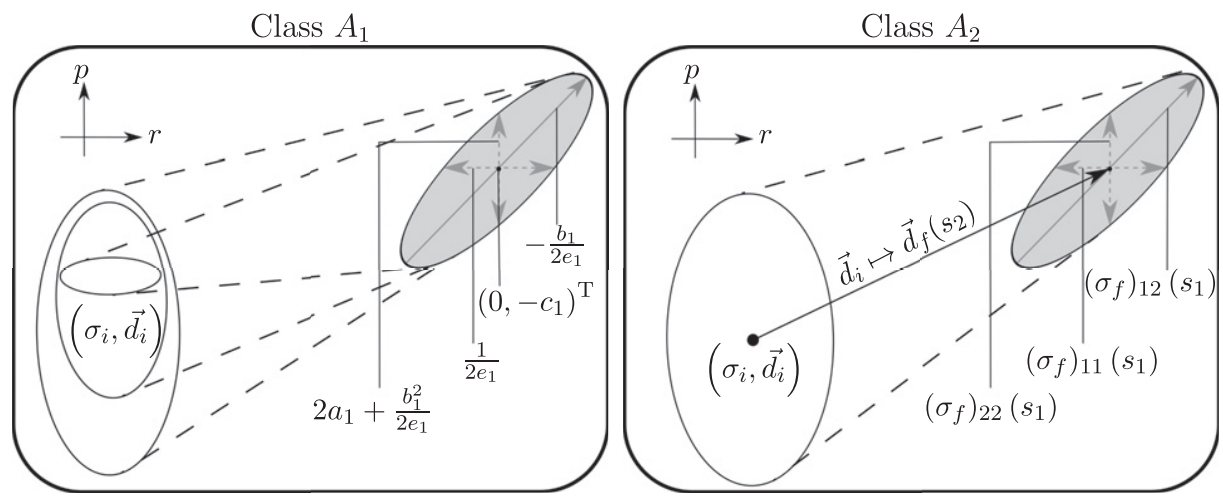

Figure 1. Schematic pictures of the channels belonging to classes $A_{1}$ and $A_{2}$ (right and left panels, respectively), acting on pictorial Wigner's functions of Gaussian states (represented with ellipses). The coordinate system corresponds to the position variable $r$ and its conjugate momentum, $p$. The figure shows how every channel in class $\mathrm{A}_{1}$ maps every initial quantum state, in particular GSs characterized by $\left(\sigma_{i}, \vec{d}_{i}\right)$, to a Gaussian state that depends only on the channel parameters. The values of the first and second moments of the final Gaussian state are indicated by a gray ellipse. Similarly, for class $\mathrm{A}_{2}$, we indicate the form of the final moments for initial Gaussian states. In this case they depend on two combinations of the initial parameters, $s_{1}$ and $s_{2}$, whose explicit formulas are given in the appendix, together with the form of the final moments.

with $e_{2}=\alpha=b_{2}=0$ and equation (27)) maps every initial state to a single one characterized by $\sigma_{\mathrm{f}}=\mathbf{N}$ and $\vec{d}_{f}=\left(0,-c_{1}\right)^{\mathrm{T}}$, see figure 1 (left panel) for a schematic description.

According to our ansätze (see equations (5) and (6)), we conclude that one-mode SGQC can only have the functional forms given in equations (3) and (5). This is one of the central results of our work and can be stated as:

Theorem 1 (One-mode singular Gaussian channels). A one-mode Gaussian quantum channel is singular if and only if it has one of the following functional forms in the position space representation

(a) $\frac{b_{3}}{2 \pi} \exp \left[\imath\left(b_{1} x_{\mathrm{f}} r_{\mathrm{f}}+b_{3} x_{\mathrm{i}} r_{\mathrm{f}}+b_{4} x_{\mathrm{i}} r_{\mathrm{i}}+c_{1} x_{\mathrm{f}}+c_{2} x_{\mathrm{i}}\right)-a_{1} x_{\mathrm{f}}^{2}-a_{2} x_{\mathrm{f}} x_{\mathrm{i}}-a_{3} x_{\mathrm{i}}^{2}\right]$,

(b) $|\beta| \sqrt{e_{1} / \pi} \delta\left(\alpha x_{\mathrm{f}}-\beta x_{\mathrm{i}}\right) \exp \left[-a_{2} x_{\mathrm{f}} x_{\mathrm{i}}-a_{1} x_{\mathrm{f}}^{2}-a_{3} x_{\mathrm{i}}^{2}\right.$

$+\imath\left(b_{2} x_{\mathrm{f}} r_{\mathrm{i}}+b_{3} r_{\mathrm{f}} x_{\mathrm{i}}+b_{1} r_{\mathrm{f}} x_{\mathrm{f}}+b_{4} r_{\mathrm{i}} x_{\mathrm{i}}+c_{1} x_{\mathrm{f}}+c_{2} x_{\mathrm{i}}\right)$

$\left.-e_{1} r_{\mathrm{f}}^{2}-e_{2} r_{\mathrm{f}} r_{\mathrm{i}}-\frac{e_{2}^{2} r_{\mathrm{i}}^{2}}{4 e_{1}}\right]$, with $e_{2} \alpha=0$.

Corollary 1 (Singular classes). A one-mode singular Gaussian channel belongs to class $\mathrm{A}_{1}$ if and only if its position representation has the following form:

$$
\sqrt{e_{1} / \pi} \delta\left(x_{\mathrm{i}}\right) \exp \left[-a_{1} x_{\mathrm{f}}^{2}+\imath\left(b_{2} x_{\mathrm{f}} r_{\mathrm{i}}+b_{1} r_{\mathrm{f}} x_{\mathrm{f}}+c_{1} x_{\mathrm{f}}\right)-e_{1} r_{\mathrm{f}}^{2}\right] .
$$

Otherwise the channel belongs to class $\mathrm{A}_{2}$.

As a particular example of theorem 1 , choosing the case (ii) with $e_{2}=2 e_{1}=(2 \bar{n}+1)^{-1}$, $\alpha=0=b_{1}=b_{2}=0, a_{1}=(2 \bar{n}+1) / 2$ one gets the Holevo's canonical form of class $\mathrm{A}_{2}$, see reference [13]. The parameter $\bar{n}$ is the average of the number of excitation in the mode. Notice that the canonical form cannot be obtained using the Gaussian functional form, while elements 
Table 1. The first and second columns show the functional forms of $J^{(1)}$ and $J^{(2)}$, respectively. The last column shows the resulting form of the concatenation of them [see Equation (28)]. See main text for symbol coding.

\begin{tabular}{lcc}
\hline$J^{(1)}$ & $J^{(2)}$ & $J^{(\mathrm{f})}$ \\
\hline$\delta_{\mathrm{A}_{2}}^{\alpha}$ & $\mathcal{G}_{\mathcal{U}}$ & $\mathcal{G}_{\mathrm{A}_{2}}$ \\
$\mathcal{G}_{\mathcal{U}}$ & $\delta_{\mathrm{A}_{2}}^{\alpha}$ & $\delta_{\mathrm{A}_{2}}^{\alpha}$ \\
$\delta_{\mathrm{A}_{2}}^{\alpha}$ & $\delta_{\mathcal{U}}$ & $\delta_{\mathrm{A}_{2}}$ \\
$\delta_{\mathcal{U}}$ & $\delta_{\mathrm{A}_{2}}$ & $\delta_{\mathrm{A}_{2}}$ \\
$\delta_{\mathrm{A}_{2}}^{e_{2}}$ & $\mathcal{G}_{\mathcal{U}}$ & $\delta_{\mathrm{A}_{2}}^{e_{2}}$ \\
$\mathcal{G}_{\mathcal{U}}$ & $\delta_{\mathrm{A}_{2}}^{e_{2}}$ & $\mathcal{G}_{\mathrm{A}_{2}}$ \\
$\delta_{\mathrm{A}_{2}}^{e_{2}}$ & $\delta_{\mathcal{U}}$ & $\delta_{\mathrm{A}_{2}}^{e_{2}}$ \\
$\delta_{\mathcal{U}}$ & $\delta_{\mathrm{A}_{2}}^{e_{2}}$ & $\delta_{\mathrm{A}_{2}}^{e_{2}}$ \\
$\mathcal{G}_{\mathcal{U}}$ & $\delta_{\mathrm{A}_{2}}^{\alpha, e_{2}}$ & $\delta_{\mathrm{A}_{2}}^{\alpha, e_{2}}$ \\
$\delta_{\mathrm{A}_{2}}^{\alpha, e_{2}}$ & $\mathcal{G}_{\mathcal{U}}$ & $\mathcal{G}_{\mathrm{A}_{2}}$ \\
$\delta_{\mathcal{U}}$ & $\delta_{\mathrm{A}_{2}}^{\alpha, e_{2}}$ & $\delta_{\mathrm{A}_{2}}^{\alpha, e_{2}}$ \\
$\delta_{\mathrm{A}_{2}}^{\alpha, e_{2}}$ & $\delta_{\mathcal{U}}$ & $\delta_{\mathrm{A}_{2}}^{\alpha, e_{2}}$ \\
$\delta_{\mathcal{U}}, \mathcal{G}_{\mathcal{U}}$ & $\delta_{\mathrm{A}_{1}}$ & $\delta_{\mathrm{A}_{1}}$ \\
$\delta_{\mathrm{A}_{1}}$ & $\delta_{\mathcal{U}}, \mathcal{G}_{\mathcal{U}}$ & $\delta_{\mathrm{A}_{1}}$ \\
\hline
\end{tabular}

in $A_{2}$ enjoy both (i) and (ii) forms of theorem 1 (see table 1). Additionally the operator sum representation of this canonical form is given in reference [10].

Since channels on each class are connected each other by unitary conjugations [11], a consequence of the theorem and the subsequent corollary is that the set of allowed forms must remain invariant under unitary conjugations. To show this we must know the possible functional forms of Gaussian unitaries. They are given by following lemma for one mode

Lemma 1 (One-mode Gaussian unitaries). Gaussian unitaries have only GF or the one given by equation (6).

Proof. Recalling that for a unitary GQC, $\mathbf{T}$ must be symplectic $\left(\mathbf{T} \Omega \mathbf{T}^{\mathrm{T}}=\Omega\right)$ and $\mathbf{N}=\mathbf{0}$. However, an inspection to equation (17) lead us to note that $\mathbf{N} \neq \mathbf{0}$ unless $e_{1}$ diverges. Thus, Gaussian unitaries cannot have the form $J_{\mathrm{I}}$ (see equation (5)). An inspection of matrices $\mathbf{T}$ and $\mathbf{N}$ of GQC with GF (see equation (26)) and the ones for $J_{\text {II }}$ (see equations (18) and (24)) lead us to note the following two observations: (i) in both cases we have $\mathbf{N}=0$ for $a_{n}=0 \forall n$; (ii) the matrix $\mathbf{T}$ is symplectic for GF when $b_{2}=b_{3}$, and when $\alpha \eta=\beta \gamma$ for $J_{\mathrm{II}}$. In particular the identity map has the last form. This completes the proof.

One can now compute the concatenations of the SGQCs with Gaussian unitaries. This can be done straightforward using the well known formulas for Gaussian integrals and the Fourier transform of the Dirac delta. Given that the calculation is elementary, and for sake of brevity, we present only the resulting forms of each concatenation. To show this compactly we introduce the following abbreviations: Singular channels belonging to class $\mathrm{A}_{2}$ with form $J_{\mathrm{I}}$ and with $\alpha=0, e_{2}=0$ and $\alpha=e_{2}=0$, will be denoted as $\delta_{\mathrm{A}_{2}}^{\alpha}, \delta_{\mathrm{A}_{2}}^{e_{2}}$ and $\delta_{\mathrm{A}_{2}}^{\alpha, e_{2}}$, respectively; singular channels belonging to the same class but with $\mathrm{GF}$ will be denoted as $\mathcal{G}_{\mathrm{A}_{2}}$; channels belonging to class $\mathrm{A}_{1}$ will be denoted as $\delta_{\mathrm{A}_{1}}$; finally Gaussian unitaries with GF will be denoted as $\mathcal{G}_{\mathcal{U}}$ and the ones with form $J_{\text {II }}$ as $\delta_{\mathcal{U}}$. Writing the concatenation of two channels in the position representation as

$$
J^{(\mathrm{f})}\left(x_{\mathrm{f}}, r_{\mathrm{f}} ; x_{\mathrm{i}}, r_{\mathrm{i}}\right)=\int_{\mathbb{R}^{2}} \mathrm{~d} x^{\prime} \mathrm{d} r^{\prime} J^{(1)}\left(x_{\mathrm{f}}, r_{\mathrm{f}} ; x^{\prime}, r^{\prime}\right) J^{(2)}\left(x^{\prime}, r^{\prime} ; x_{\mathrm{i}}, r_{\mathrm{i}}\right),
$$


the resulting functional forms for $J^{(\mathrm{f})}$ are given in table 1. As expected, the table shows that the integral has only the forms stated by our theorem. Additionally it shows the cases when unitaries change the functional form of class $\mathrm{A}_{2}$, while for class $\mathrm{A}_{1}, J^{(\mathrm{f})}$ has always the unique form enunciated by the corollary. This table also constitutes another proof for our theorem 1 and the corollary 1 .

The central results of this section are the following. The three functional forms existing for one-mode Gaussian channels in position state representation allow specific types of channels. We proved that only channels with GF or $J_{\text {II }}$ can be unitary, whereas channels with GF or $J_{\mathrm{I}}$ can be singular. To show the relevance of this result, we introduce the following example in which the transition from one functional form to another determines the change from unitarity to non-unitarity.

Example 1 (Unitary transition). Let $\delta\left(r_{\mathrm{f}}-r_{\mathrm{i}} \eta / \gamma\right) \delta\left(x_{\mathrm{f}} \gamma / \eta-x_{\mathrm{i}}\right) \exp (\Sigma)$ be a family of unitary channels with functional form $J_{\mathrm{II}}$, fulfilling equations (12) and (25) with $a_{n}=0, \forall n$. Substituting $\delta\left(r_{\mathrm{f}}-\eta / \gamma\right) \rightarrow \exp \left[-\left(r_{\mathrm{f}}-r_{\mathrm{i}} \eta / \gamma\right)^{2} / \epsilon^{2}\right] /(\epsilon \sqrt{\pi})$ with $\epsilon>0$, we recover the mentioned family of channels in the limit of $\epsilon \rightarrow 0$. However, for finite $\epsilon$ we have a family of valid channels with form $J_{\mathrm{I}}$, see equation (5). It is easy to check that equations (11) and (23) are fulfilled, the latter for $\gamma \neq 0$ and $\beta \neq 0$. Since the form $J_{\mathrm{I}}$ cannot describe unitary channels, the transition $J_{\mathrm{I}} \leftrightarrow J_{\mathrm{II}}$ coincides with the transition non-unitary/unitary.

This example shows how the regularization of delta like factors in quantum channels leads to lose key properties, such as unitarity. This motivates investigating if other quantum information properties are lost when performing regularizations.

\section{Existence of master equations}

In this section we show the conditions under which master equations, associated with the channels derived in section 3, exist. To be more precise, we prove that, under a simple condition, the functional forms derived above parametrize channels belonging to one-parameter differentiable families of GQCs. The latter, together the result given in reference [15], implies that channels with any functional form can be found in the vicinity of the identity map. As a first step to derive the condition of existence of a differentiable family, we let the coefficients of forms presented in equations (5) and (6) to depend on time. Later we derive the conditions under which they bring any quantum state $\rho(x, r ; t)$ to $\rho(x, r ; t+\epsilon)($ with $\epsilon>0$ and $t \in[0, \infty))$ smoothly, while holding the specific functional form of the channel, i.e.

$$
\rho(x, r ; t+\epsilon)=\rho(x, r ; t)+\epsilon \mathcal{L}_{t}[\rho(x, r ; t)]+\mathcal{O}\left(\epsilon^{2}\right),
$$

where both $\rho(x, r ; t)$ and $\rho(x, r ; t+\epsilon)$ are propagated from $t=0$ with channels either with the form $J_{\mathrm{I}}$ or $J_{\mathrm{II}}$, and $\mathcal{L}_{t}$ is a bounded superoperator in the state subspace. This is basically the problem of the existence of a master equation

$$
\partial_{t} \rho(x, r ; t)=\mathcal{L}_{t}[\rho(x, r ; t)],
$$

for such functional forms. Thus, the problem is reduced to prove the existence of the linear generator $\mathcal{L}_{t}$, also known as Liouvillian.

To do this we use an ansatz proposed in reference [18] to investigate the existence and derive the master equation for GFs,

$$
\mathcal{L}=\mathcal{L}_{c}(t)+\left(\partial_{x}, \partial_{r}\right) \mathbf{X}(t)\left(\begin{array}{c}
\partial_{x} \\
\partial_{r}
\end{array}\right)+(x, r) \mathbf{Y}(t)\left(\begin{array}{c}
\partial_{x} \\
\partial_{r}
\end{array}\right)+(x, r) \mathbf{Z}(t)\left(\begin{array}{l}
x \\
r
\end{array}\right)
$$


where $\mathcal{L}_{c}(t)$ is a complex function and

$$
\mathbf{X}(t)=\left(\begin{array}{ll}
X_{x x}(t) & X_{x r}(t) \\
X_{r x}(t) & X_{r r}(t)
\end{array}\right)
$$

is a complex matrix as well as $\mathbf{Y}(t)$ and $\mathbf{Z}(t)$, whose entries are defined in a similar way as in equation (32). Note that $\mathbf{X}(t)$ and $\mathbf{Z}(t)$ can always be chosen symmetric, i.e. $X_{x r}=X_{r s}$ and $Z_{x r}=Z_{r x}$. Thus, we must determine 11 time-dependent functions from equation (31). This ansatz is also appropriate to study the functional forms introduced in this work, given that the left-hand side of equation (30) only involves quadratic polynomials in $x, r, \partial / \partial x$ and $\partial / \partial r$, as in the GF case.

Notice that singular channels do not admit a master equations since its existence implies that channels with the functional form involved can be found arbitrarily close from the identity channel. This is not possible for singular channels due to the continuity of the determinant of the matrix $\mathbf{T}$.

For the non-singular cases presented in equations (5) and (6), the condition for the existence of a master equation is obtained as follows. (i) Substitute the ansatz of equation (31) in the right-hand side of the equation (30). (ii) Define $\rho(x, r ; t)$ using equation (2), given an initial condition $\rho(x, r ; 0)$, for each functional form $J_{\mathrm{I}, \mathrm{II}}$. (iii) Take $\rho_{\mathrm{f}}\left(x_{\mathrm{f}}, r_{\mathrm{f}}\right) \rightarrow \rho(x, r ; t)$ and $\rho_{\mathrm{i}}\left(x_{\mathrm{i}}, r_{\mathrm{i}}\right) \rightarrow$ $\rho(x, r ; 0)$. Finally, (iv) compare both sides of equation (30). Defining $A(t)=\alpha(t) / \beta(t)$ and $B(t)=\gamma(t) / \eta(t)$, the conclusion is that for both $J_{\mathrm{I}}$ and $J_{\mathrm{II}}$, a master equation exists if and only if $c_{1}(t) \dot{A}(t)=A(t)\left(\dot{c}_{1}(t)+A(t) c_{2}(t)\right)$, this can be easily simplified, by adding $A(t) \dot{A}(t) c_{2}(t)$ in both sides the equation and integrating respect to $t$, to

$$
c(t) \propto A(t)
$$

where $c(t)=c_{1}(t)+A(t) c_{2}(t)$. Additionally, for the form $J_{\mathrm{I}}$ the solutions for the matrices $\mathbf{X}(t)$, $\mathbf{Y}(t)$ and $\mathbf{Z}(t)$ are given by

$$
\begin{aligned}
X_{x x} & =X_{x r}=Y_{r x}=Z_{r r}=0, \\
Y_{x x} & =\frac{\dot{A}}{A}, \\
\mathcal{L}_{c} & =Y_{r r}=\frac{\dot{e}_{1}}{e_{1}}-\frac{\dot{e}_{2}}{e_{2}}, \\
X_{r r} & =\frac{\dot{e}_{1}}{4 e_{1}^{2}}-\frac{\dot{e}_{2}}{2 e_{1} e_{2}}, \\
Y_{x r} & =\imath\left(\frac{\lambda_{1} \dot{e}_{2}}{e_{1} e_{2}}+\frac{\lambda_{2} \dot{A}}{e_{2} A}-\frac{\lambda_{1} \dot{e}_{1}}{2 e_{1}^{2}}-\frac{\dot{\lambda}_{2}}{e_{2}}\right), \\
Z_{x x} & =\frac{\lambda_{1}^{2}}{2}\left(\frac{\dot{e}_{2}}{e_{1} e_{2}}-\frac{\dot{e}_{1}}{2 e_{1}^{2}}\right)+\frac{\lambda_{1}}{e_{2}}\left(\frac{\lambda_{2} \dot{A}}{A}-\dot{\lambda}_{2}\right)+2 \lambda_{3} \frac{\dot{A}}{A}-\dot{\lambda}_{3}, \\
Z_{x r} & =\imath\left(\frac{\dot{A}}{A}\left(\frac{e_{1} \lambda_{2}}{e_{2}}-\frac{\lambda_{1}}{2}\right)+\frac{\dot{\lambda}_{1}}{2}-\frac{\dot{\lambda}_{2} e_{1}}{e_{2}}+\frac{\lambda_{2}}{2}\left(\frac{\dot{e}_{2}}{e_{2}}-\frac{\dot{e}_{1}}{e_{1}}\right)\right),
\end{aligned}
$$

where we have defined the following coefficients: $\lambda_{1}=b_{1}+A b_{3}, \lambda_{2}=b_{2}+A b_{4}$ and $\lambda_{3}=a_{1}+A a_{2}+A^{2} a_{3}$. 
For the form $J_{\text {II }}$ the solutions are the following

$$
\begin{aligned}
\mathcal{L}_{c}= & X_{x x}=X_{x r}=X_{r r}=Z_{r r}=Y_{r x}=Y_{x r}=0, \\
Y_{x x}= & \frac{\dot{A}}{A}, Y_{r r}=\frac{\dot{B}}{B} . \\
Z_{x x}= & a_{2}(t) \dot{A}(t)+\frac{2 a_{1}(t) \dot{A}(t)}{A(t)}-A(t)^{2} \\
& -\dot{a}_{3}(t)-A(t) \dot{a}_{2}(t)-\dot{a}_{1}(t), \\
Z_{x r}= & \imath\left(\frac{1}{2} \dot{\lambda}-\frac{\lambda}{2}\left(\frac{\dot{A}}{A}+\frac{\dot{B}}{B}\right)\right),
\end{aligned}
$$

where $\lambda=b_{1}+A b_{3}+B\left(b_{2}+A b_{4}\right)$.

Summarizing, letting the coefficients of forms $J_{\mathrm{I}}$ and $J_{\mathrm{II}}$ depend on time to define oneparametric families, we have derived the condition for such families to be smooth, see equation (33). Additionally we have found the explicit expressions for the ansatz coefficients for both functional forms, see sets of equations (34) and (35). In particular for $J_{\text {II }}$ the generator is simpler than the one for $J_{\mathrm{I}}$; this is expected because channels with form $J_{\mathrm{II}}$ depend on less parameters than $J_{\mathrm{I}}$. Let us notice that although singular channels do not admit master equations for the reasons described above, it must not be confused with the fact that they can be reached by smooth quantum processes.

\section{Conclusions}

In this work we have critically reviewed the deceptively natural idea that Gaussian quantum channels always admit a Gaussian functional form. To this end, we went beyond the pioneering characterization of Gaussian channels with Gaussian form presented in reference [15] in two new directions. First we have shown that, starting from their most general definition as mapping Gaussian states into Gaussian states, a more general parametrization of the coordinate representation of the one-mode case exists, that admits non-Gaussian functional forms. Second, we were able to provide a black-box characterization of such new forms by imposing complete positivity (not considered in reference [15]) and trace preserving conditions. While our parametrization connects with the analysis done by Holevo [17] in the particular cases where besides having a non-Gaussian form the channel is also singular, it also allows the study of Gaussian unitaries, thus providing similar classification schemes. We completed the classification of the studied types of channels by deriving the form of the Liouvillian super operator that generates their time evolution in the form of a master equation. Surprisingly, Gaussian quantum channels without Gaussian form can be experimentally addressed by means of the celebrated Caldeira-Legget model for the quantum damped harmonic oscillator [14], where the new types of channels described here naturally appear in the sub-ohmic regime.

\section{Acknowledgments}

We acknowledge PAEP and RedTC for financial support. Support by projects CONACyT 285754, UNAM-PAPIIT IG100518 is acknowledged. CP acknowledges support by PASPA 
program from DGAPA-UNAM. CAM and JDU acknowledge financial support from the German Academic Exchange Service (DAAD). We are thankful to the University of Vienna where part of this project was done.

\section{Appendix A. Explicit formulas for class $A_{2}$}

The explicit formulas of the final states for channels of class $\mathrm{A}_{2}$ with the form presented in equation (6) with $e_{2}=0$ are

$$
\begin{aligned}
& \left(\sigma_{f}\right)_{11}=\frac{1}{2 e_{1}} \\
& \left(\sigma_{f}\right)_{22}=\left(\frac{\alpha}{\beta}\right)^{2}\left(\frac{b_{3}^{2}}{2 e_{1}}+2 a_{3}\right)+\frac{\alpha}{\beta}\left(2 a_{2}+\frac{b_{1} b_{3}}{e_{1}}\right)+2 a_{1}+\frac{b_{1}^{2}}{2 e_{1}}+s_{1}, \\
& \left(\sigma_{f}\right)_{12}=-\frac{\alpha}{\beta} \frac{b_{3}}{2 e_{1}}-\frac{b_{1}}{2 e_{1}}, \\
& \vec{d}_{f}\left(s_{3}\right)=\left(0,-\frac{\alpha}{\beta} c_{2}-c_{1}+s_{2}\right)^{\mathrm{T}},
\end{aligned}
$$

where

$$
\begin{aligned}
s_{1} & =\left(b_{2}^{2}+2 \frac{\alpha}{\beta} b_{2} b_{4}+\left(\frac{\alpha}{\beta}\right)^{2} b_{4}^{2}\right)\left(\sigma_{i}\right)_{11}-2\left(\frac{\alpha}{\beta} b_{2}+\left(\frac{\alpha}{\beta}\right)^{2} b_{4}\right)\left(\sigma_{i}\right)_{12}+\left(\frac{\alpha}{\beta}\right)^{2}\left(\sigma_{i}\right)_{22}, \\
s_{2} & =\left(\frac{\alpha}{\beta} b_{4}+b_{2}\right)\left(d_{i}\right)_{1}-\frac{\alpha}{\beta}\left(d_{i}\right)_{2} .
\end{aligned}
$$

The explicit formulas of the final states for channels of class $A_{2}$ with the form presented in equation (6) with $\alpha=0$ are

$$
\begin{aligned}
& \left(\sigma_{f}\right)_{11}=\frac{e_{2}^{2}}{4 e_{1}^{2}}\left(\sigma_{i}\right)_{11}+\frac{1}{2 e_{1}}, \\
& \left(\sigma_{f}\right)_{12}=\left(\frac{b_{2} e_{2}}{2 e_{1}}-\frac{b_{1} e_{2}^{2}}{4 e_{1}^{2}}\right)\left(\sigma_{i}\right)_{11}-\frac{b_{1}}{2 e_{1}}, \\
& \left(\sigma_{f}\right)_{22}=2 a_{1}+\left(b_{2}-\frac{b_{1} e_{2}}{2 e_{1}}\right)^{2}\left(\sigma_{i}\right)_{11}+\frac{b_{1}^{2}}{2 e_{1}},
\end{aligned}
$$

and

$$
\vec{d}_{f}=\left(\frac{e_{2}}{2 e_{1}}\left(\vec{d}_{i}\right)_{1},\left(b_{2}-\frac{b_{1} e_{2}}{2 e_{1}}\right)\left(\vec{d}_{i}\right)_{1}-c_{1}\right)^{\mathrm{T}}
$$

The explicit formulas of the final states for channels of class $A_{2}$ with Gaussian form are

$$
\begin{aligned}
& \left(\sigma_{f}\right)_{11}\left(s_{1}\right)=\frac{2 a_{3}}{b_{3}^{2}}+s_{1}, \\
& \left(\sigma_{f}\right)_{12}\left(s_{1}\right)=\frac{a_{2}}{b_{3}}-\frac{2 a_{3} b_{1}}{b_{3}^{2}}-b_{1} s_{1},
\end{aligned}
$$




$$
\begin{gathered}
\left(\sigma_{f}\right)_{22}\left(s_{1}\right)=\frac{b_{1}\left(b_{3}\left(b_{1} b_{3} s_{1}-2 a_{2}\right)+2 a_{3} b_{1}\right)}{b_{3}^{2}}+2 a_{1}, \\
\vec{d}_{f}\left(s_{2}\right)=\left(s_{2}-\frac{c_{2}}{b_{3}}, b_{1}\left(\frac{c_{2}}{b_{3}}-s_{2}\right)-c_{1}\right)^{\mathrm{T}},
\end{gathered}
$$

where

$$
\begin{aligned}
& s_{1}=\frac{b_{4}^{2}}{b_{3}^{2}}\left(\sigma_{i}\right)_{11}-\frac{2 b_{4}}{b_{3}^{2}}\left(\sigma_{i}\right)_{12}+\frac{1}{b_{3}^{2}}\left(\sigma_{i}\right)_{22}, \\
& s_{2}=\frac{1}{b_{3}}\left(d_{i}\right)_{2}-\frac{b_{4}}{b_{3}}\left(d_{i}\right)_{1} .
\end{aligned}
$$

\section{ORCID iDs}

David Davalos (1D https://orcid.org/0000-0001-8091-6120

Camilo Moreno (D) https://orcid.org/0000-0002-8969-2407

Carlos Pineda (D) https://orcid.org/0000-0002-7306-0894

\section{References}

[1] Cerf N J, Leuchs G and Polzik E S 2007 Quantum Information with Continuous Variables of Atoms and Light (London: Imperial College Press)

[2] Aspelmeyer M, Kippenberg T J and Marquardt F 2014 Cavity optomechanics Rev. Mod. Phys. 86 $1391-452$

[3] Hammerer K, Sørensen A S and Polzik E S 2010 Quantum interface between light and atomic ensembles Rev. Mod. Phys. 82 1041-93

[4] Grosshans F, Van Assche G, Wenger J, Brouri R, Cerf N J and Grangier P 2003 Quantum key distribution using Gaussian-modulated coherent states Nature $\mathbf{4 2 1} 238$

[5] Lloyd S and Braunstein S L 1999 Quantum computation over continuous variables Phys. Rev. Lett. 82 1784-7

[6] Braunstein S L and van Loock P 2005 Quantum information with continuous variables Rev. Mod. Phys. 77 513-77

[7] Lami L, Regula B, Wang X, Nichols R, Winter A and Adesso G 2018 Gaussian quantum resource theories Phys. Rev. A 98022335

[8] Martinez E A and Paz J P 2013 Dynamics and thermodynamics of linear quantum open systems Phys. Rev. Lett. 110130406

[9] Moshinsky M and Quesne C 1971 Linear canonical transformations and their unitary representations J. Math. Phys. 12 1772-80

[10] Ivan J S, Sabapathy K K and Simon R 2011 Operator-sum representation for bosonic Gaussian channels Phys. Rev. A 84042311

[11] Holevo A S 2007 One-mode quantum Gaussian channels: structure and quantum capacity Probl. Inf. Transm. 43 1-11

[12] Eisert J and Wolf M M 2007 Gaussian quantum channels Quantum Information with Continuous Variables of Atoms and Light (London: Imperial College Press) pp 23-42

[13] Weedbrook C, Pirandola S, García-Patrón R, Cerf N J, Ralph T C, Shapiro J H and Lloyd S 2012 Gaussian quantum information Rev. Mod. Phys. 84 621-69

[14] Grabert H, Schramm P and Ingold G-L 1988 Quantum Brownian motion: the functional integra approach Phys. Rep. 168 115-207

[15] Martinez E A and Paz. J P 2013 Dynamics and thermodynamics of linear quantum open systems Phys. Rev. Lett. 110130406

[16] Lindblad G 2000 Cloning the quantum oscillator J. Phys. A: Math. Gen. 33 5059-76

[17] Holevo A S 2008 Entanglement-breaking channels in infinite dimensions Probl. Inf. Transm. 44 171-84

[18] Karrlein R and Grabert H 1997 Exact time evolution and master equations for the damped harmonic oscillator Phys. Rev. E 55 153-64 